\title{
Teneur en énergie métabolisable et digestibilité des acides aminés chez le coq de deux sous-produits de l'extraction de l'huile : tourteau de palmiste pressé (Elaeis guineensis) et germe et son de maïs extrait (Zea mays)
}

\author{
M. Vilariño ${ }^{1}$ F. Rudeaux ${ }^{2}$ A. León ${ }^{1}$ M. Picard ${ }^{3}$
}

Mots-clés

Volaille - Coq - Palmiste - Maïs Germe de céréale - Son - Acide aminé - Valeur énergétique Composition chimique - Digestibilité Venezuela.

\begin{abstract}
Résumé
La digestibilité d'un échantillon de tourteau de palmiste vénézuélien (TP) [contenant 92,2 p. 100 de matière sèche (MS) et en pourcentage de MS : 13,5 de protéine brute $(P B), 14,6$ de matières grasses $(M G), 4,6$ de matières minérales $(M M), 60,7$ de parois végétales (PAR) et moins de 1 p. 100 d'amidon et de sucres] est mesurée chez neuf coqs nourris ad libitum (30 p. 100 TP + 67 p. 100 maïs témoin +3 p. 100 prémélange). La valeur d'énergie métabolisable apparente (EMA) de TP est $1963 \pm 52 \mathrm{kcal} / \mathrm{kg} \mathrm{MS}$. La digestibilité vraie moyenne des acides aminés de TP est 48 p. 100 (pour la lysine, 26 p. 100). Les mêmes mesures sont faites sur un sous-produit vénézuélien de l'extraction de l'huile et de la farine de maïs contenant les germes et du son (GSME) [85,9 p. 100 MS et en p. 100 MS : 14,1 PB, 1,3 MG, 3,9 MM, 34,2 PAR, 44,0 d'amidon et 6,2 de sucres] à 50 p. 100 dans le régime de test (+ 47 p. 100 maïs témoin +3 p. 100 prémélange). La valeur EMA de GSME est $2648 \pm 28 \mathrm{kcal} / \mathrm{kg}$ MS. La digestibilité vraie moyenne des acides aminés de GSME est de 89 p. 100 (pour la lysine, 82 p. 100). Les coefficients de digestibilité élevés pour les protéines et l'amidon de GSME facilitent la prédiction de sa valeur EM A pour les volailles à partir de l'analyse chimique. Le TP testé est essentiellement une source de lipides résiduels dont la teneur détermine la valeur nutritionnelle pour les volailles.
\end{abstract}

\section{INTRODUCTION}

Le développement de l'aviculture dans les pays tropicaux est limité par le coût en devises des matières premières importées pour la fabrication des aliments. Au Venezuela, 40 p. 100 des protéines d'origine animale consommées par la population proviennent de l'aviculture. Ce pays importe 70 p. 100 des matières premières nécessaires pour nourrir les volailles, malgré l'existence de sous-produits disponibles localement. Le principal facteur limitant une utilisation correcte des sous-produits est l'évaluation imprécise de leur valeur nutritionnelle réelle. Ils sont utilisés aujourd'hui sur la base des valeurs moyennes trouvées dans les tables internationales qui peuvent différer sensiblement de la réalité.

Le tourteau de palmiste (TP) a été étudié pour l'alimentation des volailles dans les pays tropicaux $(11,12)$. Il provient de l'industrie

1. Instituto de Investigaciones Zootécnicas, Fondo Nacional de Investigaciones Agropecuarias, Apdo. Postal 4653, Maracay 2101, Venezuela

2. Sanders Aliments, BP 32, 91201 Athis Mons Cedex, France

3. Station de recherches avicoles, INRA, 37380 Nouzilly, France de l'huile de palme africaine (Elaeis guineensis) qui débute au Venezuela. Les disponibilités actuelles de tourteau de palmiste avoisinent 10000 tonnes par an. Cependant, de 1981 à 1990 au Venezuela, la surface cultivée en palmier à huile a augmentée en moyenne de 5,6 p. 100 par an et la production des fruits de 7,7 p. 100 par an (données du Ministère de l'Agriculture du Venezuela).

Le tourteau de germe et de son de maïs obtenu par extraction (GSME) provient de la production de farine et d'huile de maïs. Plus de 20 millions de vénézuéliens consomment traditionnellement un petit pain fait avec une farine précuite qui est obtenue à partir du maïs. Les résidus de cette fabrication (germes et son) sont utilisés pour l'extraction de l'huile, ce qui donne deux produits essentiels pour la consommation humaine (la farine et l'huile) et laisse des quantités importantes de tourteaux utilisables pour l'alimentation animale (330 000 tonnes/an en 1993, selon l'entreprise Remavenca).

L'analyse chimique des matières premières, essentiellement les dosages de l'amidon, des sucres, des matières grasses, des acides aminés et des parois végétales ont une valeur prédictive de la va- 
leur nutritionnelle pour les volailles. Cependant, dans une démarche logique pour évaluer une matière première mal connue, l'analyse chimique doit être suivie de tests d'acceptabilité (ingestion/toxicité) puis d'essais de bilan digestif et d'expériences zootechniques (17). L'évaluation directe sur les animaux de la digestibilité des nutriments est une étape essentielle, spécialement pour les sous-produits encore mal identifiés et ayant subi des traitements technologiques (14).

Ce travail a pour but de mesurer la valeur nutritionnelle de deux échantillons représentatifs des sous-produits majeurs utilisables au Venezuela pour alimenter les volailles.

\section{MATERIEL ET METHODES}

\section{Matières premières}

Le tourteau de palmiste (TP) est le résidu de l'extraction par pression de l'amande contenue dans les fruits du palmier à huile (Elaeis guineensis), dont une partie de l'huile reste dans le tourteau. L'échantillon étudié a été produit par la principale entreprise productrice au Venezuela: Bananera Venezolana C.A. (Edo. Yaracuy). Après l'extraction de l'huile de palme, la noix a été récupérée. L'amande contenue dans cette noix a été broyée, tamisée puis cuite avec de l'eau et enfin pressée dans une vis sans fin pour extraire l'huile de palmiste (procédé expeller). Le tourteau de palmiste restant a été à nouveau moulu.

Le sous-produit de l'extraction du germe et du son de maïs (GSME) est issu du traitement industriel du maïs à sec (il diffère du traitement par voie humide pour l'obtention de l'amidon). Après séparation de l'endosperme, produit principal utilisé pour l'élaboration d'une farine de consommation humaine, le son et les germes ont été soumis à une trituration et chauffés pour l'extraction par solvant de l'huile de maïs. Le tourteau résultant a été toasté (pour récupérer l'hexane) et refroidi, donnant le GSME. Bien qu'il existe une variabilité certaine des procédés utilisés au Venezuela pour la production de GSME et que toutes les variantes ne soient pas publiées, l'échantillon étudié est représentatif d'une production majoritairement disponible sur le marché (10). Il a été produit par Remavenca (Edo. Aragua).

\section{Composition chimique}

Les matières premières (TP, GSME et maïs témoin) ont été analysées par Sanders Aliments pour la détermination des teneurs en matière sèche, protéine brute, matière grasse (sans hydrolyse), cellulose brute, amidon (méthode Ewers), sucres (méthode Luffshorl), parois végétales (4) et matière minérale (suivant la norme AFNOR pour chaque constituant). La cellulose brute a été dosée pour donner une référence par rapport aux mesures publiées, toutefois, seule l'évaluation réelle des parois végétales a permis d'évaluer la fraction fibreuse indigestible chez les volailles (5).

\section{Energie métabolisable apparente (EMA)}

La concentration en EMA des matières premières a été mesurée à la station de Sanders Aliments à Sourches selon une méthode de bilan digestif adaptée de la méthode européenne (3). Les mesures ont porté sur 27 coqs adultes non caecectomisés de souche ISA Brown âgés de 11 mois et logés dans des cages avec plateaux de récoltes des fientes.

La souche ISA Brown est la principale souche de ponte au Venezuela. Il s'agit d'une souche demi-lourde dont les coqs sont les plus fréquemment utilisés dans les tests de bilan digestifs car ils sont plus calmes et présentent un niveau d'ingestion supérieur aux Leghorn.
Trois régimes ont été préparés : $\mathrm{RT}=$ régime témoin $[97$ p. 100 maïs + 3 p. 100 complément minéral vitaminé $(\mathrm{CMV})]$, RTP = régime tourteau de palmiste $(67$ p. 100 maïs +30 p. 100 TP +3 p. $100 \mathrm{CMV}$ ) et RGSME = régime GSME (47 p. 100 maïs + 50 p. 100 GSME + 3 p. $100 \mathrm{CMV}$ ). Chaque régime a été consommé ad libitum par neuf coqs adultes, pendant $48 \mathrm{~h}$, puis la consommation a été réduite à 90 p. 100 de l'ingéré ad libitum, pendant une phase d'adaptation de $31 \mathrm{~h}$. La période de mesure (consommation ad libitum et collecte complète de fèces) a duré trois jours, précédée et suivie par deux périodes de jeûne : $24 \mathrm{~h}$ avant (sans collecte) et $24 \mathrm{~h}$ après (avec collecte). La consommation d'aliments et le poids des fèces ont été mesurés. Les concentrations en énergie brute (EB) des fèces collectés (conservés à $-20^{\circ} \mathrm{C}$ et lyophilisés) ainsi que celle des régimes et des matières premières utilisés ont été mesurés dans un calorimètre adiabatique. Les calculs ont été corrigés pour les variations de matière sèche et l'EMA calculée selon la formule :

$$
\mathrm{EMA}=[(\mathrm{MSI} \times \mathrm{EBA})-(\mathrm{QE} \times \mathrm{EBF})] / \mathrm{MSI}
$$

où, MSI = matière sèche ingérée $; \mathrm{EBA}=$ énergie brute de l'aliment ; $\mathrm{EBF}=$ énergie brute des fèces et $\mathrm{QE}=$ quantité des fèces excrétés.

L'EMA des matières premières a été calculée pour chaque coq par différence entre l'apport énergétique total de l'aliment et l'apport provenant de la portion de maïs inclus dans chaque régime.

\section{Concentration et coefficient de digestibilité vraie des acides aminés (DVA)}

Les fèces des coqs de chaque régime, issus de la détermination d'EMA, ainsi qu'un échantillon des matières premières ont été utilisés pour la détermination du profil d'acides aminés. Un mélange de quantités aliquotes des fèces excrétés a été réalisé avant l'analyse. Les acides aminés ont été séparés sur colonne échangeur d'ions après deux hydrolyses acides de $24 \mathrm{~h}$, l'une directe et l'autre après oxydation (8). Le tryptophane a été dosé par chromatographie liquide à haute pression après hydrolyse par de la soude. Les coefficients de digestibilité apparente (DAA) et vraie (DVA) de chaque acide aminé ont été calculés selon les formules :

$$
\text { DAA }=(\text { QAAcons - QAAexc }) / \text { QAAcons }
$$

$$
\mathrm{DVA}=[\mathrm{QAAcons}-(\mathrm{QAAexc}-\mathrm{QAAend})] / \mathrm{QAAcons}
$$

où, QAAcons = quantité consommée de l'acide aminé $;$ QAAexc = quantité totale excrétée de l'acide aminé ; QAAend = quantité excrétée de l'acide aminé d'origine endogène. Cette dernière a été estimée en considérant que chaque coq élimine quotidiennement 300 mg d'un ensemble d'acides aminés d'origine endogène (13). Afin de limiter l'imprécision induite par cette approximation et pour faciliter les comparaisons avec d'autres travaux, les valeurs de digestibilité apparente sont également présentées. Cependant, compte tenu de l'incidence des excrétions endogènes d'acides aminés sur le bilan digestif, les valeurs de digestibilité corrigées pour les pertes endogènes (DVA) donnent l'estimation la plus proche de la réalité (13).

Les mesures individuelles d'EMA ont permis de calculer la moyenne et l'écart type pour chaque matière première.

\section{- RESU LTATS ET DISCUSSION}

Deux coqs malades ont été éliminés des calculs (l'un consommant le régime témoin RT et l'autre consommant le régime RTP) ce qui a ramené les effectifs de ces deux traitements à huit coqs individuels. 


\section{Tourteau de palmiste}

L'échantillon de tourteau de palmiste analysé présentait une concentration élevée d'huile résiduelle (14,6 p. $100 \mathrm{MS}$ de matières grasses, tableau I). Les parois végétales (PAR) de cette matière première représentaient 60,7 p. $100 \mathrm{du}$ poids sec. Cette mesure, très supérieure aux 30 p. 100 de cellulose brute, représentait pour les volailles la fraction fibreuse indigestible contenue dans la matière première (5).

\section{Tableau I}

Composition chimique et énergie métabolisable apparente $(E M A)^{1}$ du maïs témoin, tourteau de palmiste pressé et du germe et son de maïs extrait

\begin{tabular}{|c|c|c|c|}
\hline $\begin{array}{l}\text { Composition } \\
\text { chimique }\end{array}$ & Maïs & $\begin{array}{l}\text { Tourteau de } \\
\text { palmiste pressé }\end{array}$ & $\begin{array}{c}\text { Germe } \\
\text { et son } \\
\text { de maïs } \\
\text { extrait }\end{array}$ \\
\hline $\begin{array}{l}\text { M atière sèche } \\
\text { (MS) \% }\end{array}$ & 86,62 & 92,25 & 85,93 \\
\hline $\begin{array}{l}\text { Protéine brute } \\
\text { (PB) \% MS }\end{array}$ & 10,15 & 13,50 & 14,07 \\
\hline $\begin{array}{l}\text { Matière grasse } \\
\text { (MG) \% MS }\end{array}$ & 4,11 & 14,55 & 1,30 \\
\hline $\begin{array}{l}\text { Matière minérale } \\
(M M) \% M S\end{array}$ & 1,47 & 4,50 & 3,92 \\
\hline $\begin{array}{l}\text { Amidon } \\
\text { (A) \% MS }\end{array}$ & 69,79 & 0,29 & 43,97 \\
\hline $\begin{array}{l}\text { Sucres } \\
\text { (S) \% MS }\end{array}$ & 1,51 & 0,50 & 6,18 \\
\hline $\begin{array}{l}\text { Cellulose brute } \\
\text { (CB) \% MS }\end{array}$ & 3,13 & 30,00 & 7,05 \\
\hline $\begin{array}{l}\text { Parois végétales } \\
\text { (PAR) \% MS }\end{array}$ & 11,89 & 60,65 & 34,21 \\
\hline $\begin{array}{l}\text { EMA } \\
(\text { kcal/kg MS })^{2}\end{array}$ & $3796 \pm 13$ & $1963 \pm 52$ & $2648 \pm 28$ \\
\hline $\begin{array}{l}\text { EM/E brute } \\
(\%)\end{array}$ & 83,96 & 36,69 & 61,14 \\
\hline \multicolumn{4}{|c|}{$\begin{array}{l}\text { EM calculé à partir de formules de prédiction pour aliments } \\
\text { complets (kcal/kg de MS) }\end{array}$} \\
\hline $\begin{array}{l}\text { EM Sibbald } \\
\text { et coll. }(16)^{3}\end{array}$ & 3595 & 1647 & 2619 \\
\hline EM Härtel $(9)^{4}$ & 3555 & 1631 & 2554 \\
\hline
\end{tabular}

1. Méthode européenne de détermination de EM in vivo.

2. Moyennes de 8 à 9 coqs \pm écarts types par traitement.

3. $\mathrm{EM}(\mathrm{kcal} / \mathrm{kg} \mathrm{MS})=(35,2 \times \mathrm{PB})+(78,5 \times \mathrm{MG})+(41 \times \mathrm{A})+(35,5 \times \mathrm{S})$

4. $\mathrm{EM}(\mathrm{kcal} / \mathrm{kg} \mathrm{MS})=(36,1 \times \mathrm{PB})+(76,9 \times \mathrm{MG})+(40,6 \times \mathrm{A})+(26,1 \times \mathrm{S})$

La valeur mesurée en EMA (1 $963 \mathrm{kcal} / \mathrm{kg}$ MS) était supérieure à celle calculée à partir de deux équations de prédiction d'usage courant pour l'estimation des régimes complets [1 647 et $1631 \mathrm{kcal} / \mathrm{kg}$; respectivement, Sibbald (16) et Härtel (9)]. Cette divergence peut être due, soit à une extraction incomplète des matières grasses pour l'analyse chimique, soit parce que la valeur
d'EMA n'est pas corrigée pour un bilan azoté nul. Cependant, chez le coq adulte nourri ad libitum, les bilans azotés sont le plus souvent proches de zéro, donc les valeurs de EMAn et EMA sont très voisines (6). Le risque d'une récolte incomplète de fèces due aux effets de la matière première riche en fibres sur le transit digestif ne peut être complètement écarté bien que la récolte dure $24 \mathrm{~h}$ après la fin de la période de consommation. La valeur de $1963 \mathrm{kcal} / \mathrm{kg}$ MS doit être considérée comme une valeur maximale d'autant que les protéines sont très mal digérées.

Les protéines (13,5 p. $100 \mathrm{MS}$ ) et acides aminés (tableau II) du tourteau de palmiste ont une digestibilité vraie très faible (48 p. 100 pour l'ensemble des acides aminés et 26 p. 100 pour la lysine). Cela pourrait être dû, soit au traitement thermique, soit aux protéines de cette matière première liées aux parois végétales dont la digestibilité est presque nulle chez les volailles (5).

La richesse en fibres végétales du tourteau de palmiste conduit à s'interroger sur leurs effets éventuels sur l'excrétion endogène d'acides aminés. En effet, la digestion d'un aliment riche en fibre pourrait augmenter la desquamation intestinale et les sécrétions digestives, ce qui pourrait accentuer l'élimination d'acides aminés d'origine endogène. L'analyse des fientes ne permet pas de distinguer l'origine des acides aminés excrétés et cet effet pourrait fausser l'évaluation de la digestibilité du tourteau de palmiste. Cependant, cette hypothèse ne semble pas vérifiée chez le coq par les travaux mesurant l'effet des fibres sur l'excrétion endogène avec des régimes protéiprives (7). L'addition de 12 p. 100 d'enveloppes de maïs broyées ou de cellulose semi-purifiée n'augmente pas l'excrétion endogène de coqs de même type génétique que ceux de la présente étude. La proportion de fibres dans les régimes contenant 30 p. 100 de tourteau de palmiste (tout comme celle de l'aliment contenant 50 p. 100 de GSME) est d'environ 15 p. 100 , donc peu différente des 12 p. 100 testés par Green (7). Toutefois, la méthode de gavage utilisée par cet auteur (indispensable pour les régimes protéiprives) limite l'ingestion à $50 \mathrm{~g}$, ce qui est susceptible de limiter les effets quantitatifs des fibres. En l'absence de preuve contraire il est donc difficile d'attribuer aux fibres la faible digestibilité des acides aminés observée dans le présent essai.

Avec des régimes riches en fibres, une fermentation accrue des bactéries des caeca peut être, sur des coqs intacts, responsable d'une légère diminution de l'excrétion endogène d'acides aminés avec des aliments protéiprives (7). L'effet de ces fermentations microbiennes existe chez des volailles de production. Comme dans le cas des fibres, l'influence des caeca pourrait alimenter une discussion sur les valeurs correctives utilisées pour la transformation de la digestibilité apparente en digestibilité «vraie» (tableau II). Les pertes endogènes constituent, comme l'indigestible, une perte pour l'animal et avec des coqs nourris ad libitum les corrections pour l'endogène ont un poids relatif beaucoup plus faible qu'avec des animaux gavés. Les mesures réalisées suggèrent donc que les protéines du tourteau de palmiste sont peu digestibles et qu'il serait illusoire, dans le cas de l'échantillon étudié, de formuler un aliment sur la base des teneurs des tables en acides aminés totaux.

Les résultats de ce travail diffèrent de ceux rapportés par Onwudike (12) qui a obtenu une valeur moyenne du TP en EMA de $2652 \mathrm{kcal} / \mathrm{kg}$ de matière sèche et une digestibilité vraie des acides aminés de 83 p. 100 en moyenne et de 89 p. 100 pour la lysine. Le calcul de l'EMA à partir des résultats de composition chimique publiés par cet auteur $(\mathrm{PB}=19$ p. $100 ; \mathrm{MG}=8$ p. 100) permet une estimation d'environ $1300 \mathrm{kcal} / \mathrm{kg} \mathrm{MS}$, ce qui suggère une surévaluation de l'EMA plus marquée encore que dans la présente étude. La mesure de digestibilité des acides aminés a été réalisée par Onwudike sur un tourteau dont la teneur en cellulose brute publiée était de 11,2 p. 100 (c'est-à-dire inférieure à celle de 
Tableau II

Concentrations moyennes en acides aminés et coefficients de digestibilité (en \%) apparente (DAA) et vraie (DVA) chez le coq adulte des matières premières évaluées

(Analyses d'acides aminés sur matières premières et échantillons de fientes regroupés de 8 ou 9 coqs par matière première)

\begin{tabular}{|c|c|c|c|c|c|c|c|c|c|}
\hline \multirow[b]{3}{*}{ Acides aminés } & \multicolumn{3}{|c|}{ Maïs } & \multicolumn{3}{|c|}{ Tourteau de palmiste } & \multicolumn{3}{|c|}{ Germe et son de maïs } \\
\hline & \multirow{2}{*}{$\%$ MS } & \multicolumn{2}{|c|}{ Digestibilité } & \multirow[t]{2}{*}{$\%$ MS } & \multicolumn{2}{|c|}{ Digestibilité } & \multirow[t]{2}{*}{$\%$ MS } & \multicolumn{2}{|c|}{ Digestibilité } \\
\hline & & DAA & DVA & & DAA & DVA & & DAA & DVA \\
\hline Acide aspartique & 0,65 & 81 & 89 & 0,94 & 46 & 50 & 1,02 & 83 & 88 \\
\hline Thréonine & 0,37 & 79 & 88 & 0,34 & 43 & 49 & 0,54 & 76 & 80 \\
\hline Sérine & 0,48 & 86 & 93 & 0,43 & 52 & 58 & 0,65 & 85 & 90 \\
\hline Acide glutamique & 1,97 & 91 & 95 & 2,31 & 54 & 57 & 2,11 & 90 & 93 \\
\hline Proline & 0,89 & 92 & 95 & 0,42 & 28 & 33 & 0,95 & 89 & 91 \\
\hline Glycine & 0,37 & 74 & 91 & 0,55 & 36 & 44 & 0,67 & 80 & 88 \\
\hline Alanine & 0,72 & 89 & 94 & 0,49 & 41 & 46 & 0,86 & 86 & 89 \\
\hline Cystine & 0,20 & 84 & 94 & 0,13 & 25 & 35 & 0,30 & 87 & 92 \\
\hline Valine & 0,50 & 86 & 92 & 0,66 & 56 & 59 & 0,71 & 85 & 89 \\
\hline Méthionine & 0,20 & 89 & 94 & 0,26 & 65 & 68 & 0,28 & 90 & 93 \\
\hline Isoleucine & 0,36 & 86 & 93 & 0,46 & 46 & 50 & 0,45 & 85 & 89 \\
\hline Leucine & 1,29 & 93 & 96 & 0,82 & 54 & 57 & 1,16 & 90 & 92 \\
\hline Tyrosine & 0,42 & 88 & 93 & 0,31 & 46 & 51 & 0,44 & 85 & 87 \\
\hline Phénylalanine & 0,48 & 89 & 94 & 0,50 & 56 & 59 & 0,57 & 89 & 90 \\
\hline Lysine & 0,28 & 70 & 82 & 0,23 & 16 & 26 & 0,61 & 77 & 82 \\
\hline Histidine & 0,29 & 90 & 94 & 0,14 & 27 & 33 & 0,38 & 89 & 92 \\
\hline Arginine & 0,45 & 87 & 94 & 1,09 & 72 & 74 & 0,94 & 91 & 94 \\
\hline Tryptophane & 0,08 & 75 & 87 & 0,12 & 38 & 44 & 0,16 & 82 & 76 \\
\hline Total & 10,00 & 85 & 92 & 10,20 & 44 & 48 & 12,80 & 85 & 89 \\
\hline
\end{tabular}

l'échantillon de TP étudié) et avec une méthode utilisant un marqueur pour détecter le début et la fin de la brève émission des fèces $(4 \mathrm{~h})$, ce qui pouvait altérer la précision. Enfin, l' «extractif non azoté» mentionné par cet auteur n'a aucun sens en nutrition dans un produit dépourvu d'amidon. Les tables internationales (15) proposent une valeur d'EMA du TP de $1490 \mathrm{kcal} / \mathrm{kg}$ MS pour un tourteau extrait au solvant, donc presque dépourvu de matières grasses (1,7 p. $100 \mathrm{MS})$. Cette estimation semble trop élevée pour un tourteau qui apporte essentiellement des protéines peu digestibles et des fibres végétales.

Ogbonna et coll. (11) ont étudié la valeur nutritionnelle de trois échantillons différents de tourteau de palmiste. L'un d'entre eux présentait une composition chimique très voisine de celle de TP $(\mathrm{PB}=14,2$ p. $100 \mathrm{MS}, \mathrm{MG}=13,5$ p. $100 \mathrm{MS}$ et $\mathrm{CB}=24,7$ p. 100 MS). Ils ont mesuré sur des poulets âgés de 44 jours une digestibilité apparente des protéines de 65 p. 100 pour un régime contenant 40 p. 100 de ce tourteau. La farine de poisson, autre constituant protéique du régime, ayant une digestibilité élevée (environ 80 p. 100), la faible digestibilité du mélange peut être attribuée au tourteau de palmiste. Un calcul approximatif effectué à partir des données publiées par ces auteurs indiquerait une digestibilité moyenne des protéines du TP d'environ 20 à 30 p. 100 .

\section{Sous-produit du germe et du son de maïs extrait}

Le GSME présentait des concentrations en protéines brutes (14,1 p. 100 MS, tableau I) et en acides aminés (tableau II) supé- rieures à celle du maïs entier. La concentration élevée en paroi végétales (34,2 p. $100 \mathrm{MS})$ de GSME était compensée par une teneur élevée en amidon (44 p. $100 \mathrm{MS}$ ) et en sucres (6,2 p. $100 \mathrm{MS}$ ).

La concentration en parois végétales de GSME a réduit la fraction digestible par rapport à une céréale et seulement 61 p. 100 de l'énergie brute de ce sous-produit ont été métabolisés chez le coq (EMA = $2648 \mathrm{kcal} / \mathrm{kg}$ MS), provenant essentiellement de l'amidon. La concentration d'EMA mesurée était comparable à celles calculées à partir des équations de prédiction pour les aliments composés [2 619 et $2554 \mathrm{kcal} / \mathrm{kg}$ MS ; respectivement, Sibbald et coll. (16) et Härtel (9)]. La digestibilité vraie des acides aminés (89 p. 100) était voisine de celle de la céréale (92 p. 100). Le régime contenant GSME présentait un taux de fibres voisin de celui contenant TP et la digestibilité des acides aminés était cependant élevée dans les mêmes conditions de mesure, ce qui suggère que la teneur en paroi végétale n'est pas responsable d'erreurs importantes dans l'évaluation de l'excrétion endogène d'acides aminés.

Il est difficile de trouver des travaux publiés étudiant un GSME correspondant exactement à la production assez spécifique du Venezuela. Barbieri et Casiraghi (1) ont étudié un sous-produit de germe de maïs extrait provenant de certains pays européens où la moitié du tourteau de germes de maïs était issu d'un traitement à sec (Italie et Allemagne $=30000$ tonnes/an chacun en 1979). Le produit analysé contenait en moyenne 17,8 p. 100 MS de protéines brutes digestibles à 75 p. 100 (digestibilité mesurée in vitro), 1,5 p. 100 MS de matière grasse et 6,1 p. 100 MS de cellulose 
brute. Cependant, le schéma de traitement industriel indiqué par ces auteurs semble légèrement différent par les conditions de préparation des germes avant l'extraction à l'hexane (floconnage et humidification) de celui utilisé pour l'obtention du GSME étudié (trituration et chauffage avec vapeur).

Le sous-produit de germe de maïs analysé par Blessin et coll. (2) est plus concentré en protéines (25 p. 100) que le GSME vénézuélien mais avec un profil d'acides aminés assez proche de celui rapporté du tableau II (si on les compare en g d'acides aminés/100 g d'acides aminés). Toutefois, l'introduction de son dans des proportions différentes de GSME (et non précisées) limite les comparaisons possibles.

Les deux sous-produits analysés dans cet article caractérisent deux situations distinctes fréquemment rencontrées dans l'étude des sous-produits tropicaux. Le TP est une matière première courante pour laquelle beaucoup de mesures existent. Les teneurs moyennes en fibres végétales et en huile varient cependant considérablement d'une publication et d'une table à l'autre. La variabilité du procédé industriel induit sans doute des traitements thermiques variables en intensité qui rendent les acides aminés plus ou moins indigestibles et il est difficile de prédire la valeur nutritionnelle de ce tourteau pour les volailles sans une détermination précise de cette digestibilité. Dans l'échantillon analysé, seuls les lipides restant à l'issue d'une extraction incomplète constituent une source d'énergie significative pour l'aviculture.

L'autre exemple est celui d'un sous-produit issu d'une industrie agro-alimentaire locale qui a développé ses propres procédés : le GSME. Dans ce cas, si cette étude préliminaire montre que l'échantillon analysé est très bien digéré par les volailles et qu'une analyse d'amidon et d'azote pourrait suffire à caractériser sa valeur nutritionnelle, il reste néanmoins à vérifier la représentativité de l'échantillon testé et la variabilité existant d'une usine à l'autre. Plusieurs études de l'Université centrale du Venezuela (10) suggèrent que cette variabilité n'est pas considérable si on se réfère aux teneurs en protéines brutes (de 13,7 $\pm 0,7$ p. 100 MS à 15,4 $\pm 0,4$ p. $100 \mathrm{MS}$ entre usines extrêmes) et cellulose brute (de 6,3 $\pm 0,4$ p. 100 MS à 7,4 $\pm 0,3$ p. 100 MS entre usines extrêmes). Compte tenu de la production de plus de 300000 tonnes/an de ce sous-produit, la poursuite des mesures de digestibilité sur des échantillons très différents permettrait de valider des données utilisables en formulation pratique.

La plupart des pays tropicaux produisent des quantités importantes de sous-produits des céréales consommées par l'homme qui représenteraient des ressources déterminantes pour l'essor de productions avicoles mieux intégrées à l'économie nationale, si leur valeur nutritionnelle était exactement évaluée (14).

Les équations de prédiction de la valeur énergétique utilisées ici s'appliquent-elles à des matières premières pures ? Le choix des deux équations se justifie par le fait que les coefficients s'appliquent aux principaux nutriments réellement utilisés par les volailles comme sources d'énergie. Ainsi les équations de Sibbald et coll. et Härtel, dont les coefficients diffèrent peu, représentent une transformation logique de l'analyse chimique de l'aliment en termes énergétiques. Cette propriété n'est pas généralisable à l'ensemble des équations de prédictions dont beaucoup sont établies pour une matière première et utilisent des termes indirects comme la teneur en fibres ou en matières minérales qui ne sont pas des nutriments énergétiques pour les volailles. Les aliments testés ici ne s'éloignent pas beaucoup de ce que serait un aliment complet pour le coq adulte dont les besoins protéiques et minéraux sont réduits à l'entretien. La difficulté des sous-produits est qu'il n'existe pas d'équations spécifiques fiables établies sur un nombre suffisant d'échantillons de caractéristiques précisément établies. Les résultats de cette étude montrent que lorsqu'une analyse chimique complète est pratiquée, les teneurs en protéines, amidon, sucres et matières grasses permettent une prédiction acceptable des valeurs d'énergie métabolisable. La comparaison entre la mesure directe de la valeur énergétique d'une matière première et celle déduite de sa composition chimique est un exercice indispensable à la validation des techniques de mesure de la digestibilité.

Les méthodes in vivo de bilan digestif mesurent la valeur nutritionnelle réelle des deux sous-produits mais elles sont lourdes et coûteuses pour être utilisées couramment. Néanmoins, l'emploi des valeurs moyennes trouvées dans des tables ou mesurées sur des produits «semblables», entraîne un risque majeur d'erreur dans la formulation des rations. La valeur nutritionnelle du TP pour les volailles semble essentiellement liée aux lipides résiduels qu'il contient et inversement proportionnelle à sa teneur en fibres végétales car les acides aminés semblent peu digestibles. Le traitement d'extraction de l'huile de palmiste peut donc faire sensiblement varier l'intérêt pratique du tourteau dans l'alimentation des volailles. Le GSME a, par contre, une digestibilité élevée des acides aminés et sa concentration énergétique semble correspondre aux teneurs analytiques en amidon, matières grasses, sucres et protéines.

\section{Remerciements}

Nous remercions Sanders Aliments et Rhône Poulenc Animal Nutrition pour leur aide essentielle dans la réalisation scientifique de ces mesures et pour leur compréhension des problèmes de nutrition des volailles sous d'autres latitudes.

\section{BIBLIO GRAPHIE}

1. BARBIERI R., CASIRAGHI E.M., 1983. Production of a food grade flour from defatted corn germ meal. J. Feed Sci.Technol., 18: 35-41.

2. BLESSIN C.W., GARCIA W.J. DEATHERAGE W.L., CAVINS J.F., IN GLETT G.E., 1973. Composition of three food products containing defatted corn germ flour. J. Feed Sci. Technol., 38: 3326.

3. BOURDILLON A., CARRE B., CONAN L., DUPERRAY J., HUYGHEBAERT G., LECLERCQ B., LESSIRE M., MCNAB J., WISEM AN J., 1990. European reference method for the in vivo determination of metabolisable energy with adult cockerels: Reproductibility, effect of food intake and comparison with individual laboratory methods. Br. Poult. Sci., 31: 557-565.

4. CARRE B., BRILLOUET J.M., 1989. Determination of water-insoluble cell walls in feeds: inter-laboratory study. J.A.O .A.C., 72: 463-467.

5. CARRE B., LECLERQ B., 1985. Digestion of polysaccharides, protein and lipids by adult cockerels fed on diets containing a pectic cell-wall material from white lupin (Lupinus albus L) cotyledon. Br. J. Nutr., $\mathbf{5 4}$ 669-680.

6. CARRE B., ROZO E., 1990. La prédiction de la valeur énergétique des matières premières destinées à l'aviculture. Prod. Anim., 3 (3) : 163-169.

7. GREEN S., 1988. Effect of dietary fibre and caecectomy on the excretion of endogenous amino acids from adult cockerels. Br. Poult. Sci., 29: 419-429.

8. GREEN S., BERTRAND S.L., DURON M.J.C., MAILLARD R., 1987. Digestibilities of aminoacids in maize, wheat and barley meals, determined with intact and caecectomized cockerels. Br. Poult. Sci., 28: 631-641.

9. HÄRTEL von H., 1977. Beziehungen zwischen der N-Korrigierten umsetzbaren Energie und den $\mathrm{N}$ ährstoffgehalten des Futters beim Huhn. Arch. Geflügelk , 41: 152-181. 
10. NUÑEZ M.A., 1993. Utilizacion de algunos subproductos agroindustriales del maiz en la alimentacion de aves. Rapport de maestria, Universidad Central de Venezuela, Maracay, Venezuela, $31 \mathrm{p}$.

11. OGBONNA J.U., LONGE O.G., LEGEL S., 1988. Investigations of the digestibility of proximate composition of commercial palm kernel meal in broiler chicks rations. Arch. Anim. Nutr., 38: 215-219.

12. ONWUDIKE O.C., 1986. Palm kernel meal as a feed for poultry. 1. Composition of palm kernel meal and availability of its amino acids to chicks. Anim. Feed Sci. Technol., 16: 179-186.

13. PICARD M., BOURDON D., LE DIVIDICH J., 1984. Methods of estimating digestibility and metabolism of crop residues and agro-industrial by-products in monogastric species in developing countries. In: Proc. FAO/ILCA, Better utilization of crop residues and by-products in animal feeding: research guidelines, Addis Ababa, Ethiopia, 5-9 March 1984 Rome, Italy, FAO, p. 163-184. (Animal production and health, No. 50).

\section{Summary}

Vilariño M., Rudeaux F., León A., Picard M. Metabolisable energy and digestible amino acids contents in cockerels of two oil extracted by-products: palm kernel meals (Elaeis guineensis) and germ-bran extracted maize (Zea mays)

Digestibility of one Venezuelan palm kernel meal (PKM) sample [containing $92.2 \%$ dry matter (DM), $13.5 \%$ DM crude protein $(C P), 14.6 \% D M$ fat $(F), 4.6 \%$ DM ashes $(A)$, $60.7 \%$ DM cell wall fibre $(P)$ and less than $1 \%$ starch and sugar] was measured in nine cockerels fed ad libitum (30\% PKM $+67 \%$ of a control maize $+3 \%$ premix). The measured metabolisable energy content (ME) of PKM was $1963 \pm 52$ $\mathrm{kcal} / \mathrm{kg} \mathrm{DM}$. The average true digestibility of amino acids from PKM was $48 \%$ (26\% for lysine). O ne sample of a Venezuelan common maize by-product resulting from flour and oil extraction and made of germs and bran (BPM) [85.9\% $D M$, and in percentage of DM: $14.1 \mathrm{CP}, 1.3 \mathrm{~F}, 3.9 \mathrm{~A}, 34.2 \mathrm{P}$, 44.0 starch and 6.2 sugar] was similarly tested with a $50 \%$ BPM inclusion level in the diet (+ control maize $47 \%$ and premix $3 \%$ ). The measured ME content of BPM was $2648 \pm$ $28 \mathrm{kcal} / \mathrm{kg} \mathrm{DM}$. The real average digestibility of amino acids of BPM was $89 \%$ ( $82 \%$ for lysine). High digestibility of protein and starch in BPM helps predict its nutritional value for chickens through chemical analyses. The tested PKM was essentially a source of residual fat, whose content determines its nutritional value for poultry.

Key words: Poultry - Cock - Palm kernel - Maize - Cereal germ - Bran - Amino acid - Energy value - Chemical composition - Digestibility - Venezuela.
14. PICARD M., LEON A., 1990. Méthodes d'évaluation et de contrôle des matières premières. In : Sauveur B. ed., L'aviculture en M éditerrannée, Colloque du Centre international de hautes études agronomiques méditerranéennes, Belgrade, Yougoslavie, 5-7 novembre 1987. Paris, France, CIHEAM, p. 71-79. (Séminaire méditerranéen $n^{\circ} 7$, Série A)

15. RHONE POULENC ANIMAL NUTRITION, 1993. Feed formulation guide, 6 th ed. Antony, France, Rhône Poulenc Animal Nutrition, $39 \mathrm{p}$.

16. SIBBALD I.R., CZARNOCKI J., SLINGER J., ASHTON G., 1963. The prediction of the metabolizable energy content of poultry feedingstuffs from a knowledge of their chemical composition. Poult. Sci., 42: 486-492

17. WALDROUP P.W.., 1982. Influence of environmental temperature on protein and amino acid needs of poultry. Feed Proc., 41 (11): 2821-2823.

Reçu le 24.10.96, accepté le 18.12.96

\section{Resumen}

Vilariño M., Rudeaux F., León A., Picard M. Contenido en energía metabolizable y digestibilidad de los amino ácidos en el gallo, de dos sub productos extraídos de aceite: torta de palmito prensado (Elaeis guineensis) y germen y afrecho de extracto de maíz (Zea mays)

Se midió la digestibilidad de una muestra de torta de palmito venezolano (TP) [conteniendo 92,2 p. 100 de materia seca (MS) y, en porcentaje de MS: 13,5 de proteína bruta (PB), 14,6 de grasas (MS), 4,6 de minerales (MM ), 60,7 de paredes vegetales (PAR) y menos de 1 p. 100 de almidones y azúcares] en nueve gallos alimentados ad libitum (30 p. 100 TP + 67 p. 100 de maíz control + 3 p. 100 de pre mezcla). El valor de la energía metabolizable aparente (EMA) de la TP es de $1963 \pm 52 \mathrm{kcal} / \mathrm{kg} \mathrm{MS}$. La digestibilidad real promedio de los amino ácidos de la TP es de 48 p. 100 (26 p. 100 para la lisina). Se realizaron las mismas medidas en un sub producto venezolano, a partir de extractos de aceite y de harina de maíz, conteniendo los gérmenes y el afrecho (GSME) $[85,9 \mathrm{p}$. 100 MS y en porcentaje de MS: 14,1 PB, 1,3 MG, 3,9 M M, 34,2 PAR, 44,0 de almidón y 6,2 de azúcares] a 50 p. 100 del régimen de prueba ( +47 p. 100 maíz control +3 p. 100 pre mezcla). El valor EMA de la GSME es de $2648 \pm 28$ $\mathrm{kcal} / \mathrm{kg}$ MS. La digestibilidad real promedio de los amino ácidos de GSME es de 89 p. 100 (82 p. 100 para la lisina). Los altos coeficientes de digestibilidad para las proteínas y el almidón de la GSME facilitan la predicción del valor EMA para las aves, a partir del análisis químico. La TP examinada es esencialmente una fuente de lípidos residuales, cuyo contenido determina el valor nutricional para las aves.

Palabras clave : Ave de corral - Gallo - Almendra de palma Maíz - Germen de cereal - Salvado - Aminoácido - Valor energético - Composición química - Digestibilidad Venezuela. 